\title{
MedChemComm
}

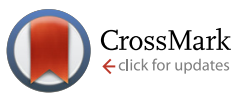

Cite this: Med. Chem. Commun., $2016,7,2184$

Received 7th July 2016,

Accepted 2nd September 2016

DOI: $10.1039 / c 6 m d 00375 c$

www.rsc.org/medchemcomm

\section{Chemical epigenetics to assess the role of HDAC1-3 inhibition in macrophage pro-inflammatory gene expression $\uparrow t$}

\author{
Maria E. Ourailidou, ${ }^{a}$ Niek G. J. Leus, ${ }^{a}$ Kim Krist, ${ }^{a}$ Alessia Lenoci, ${ }^{b}$ \\ Antonello Mai*bc and Frank J. Dekker*a
}

\begin{abstract}
Histone deacetylases (HDACs) have been used as pharmacological targets for the treatment of various diseases. Some non-selective HDAC inhibitors (HDACi) have been clinically-used as therapeutic agents for treatment of hematological cancers but their cytotoxic side effects are an important downside. The discovery of more selective inhibitors has certified the involvement of individual HDACs in pathological processes but the elucidation of the role of specific family members in inflammatory responses still remains a challenge. Here, we report the development of closely related, structural analogues of the clinically-used HDACi Entinostat via a chemical epigenetic approach. Three compounds were designed and synthesized in which the cap moiety of Entinostat was replaced by an azobenzene group that is either para, meta or ortho substituted. The compounds were then evaluated for selectivity towards HDACs 1-3 and their effect on pro-inflammatory gene expression in macrophages. One analogue, compound 4, lacked selectivity and demonstrated inhibition of NF-kB reporter gene activity and pro-inflammatory gene expression in RAW264.7 macrophages, thus indicating that there is a delicate balance between the selectivity of $\mathrm{HDACi}$ over specific family members and their pro- or anti-inflammatory effects.
\end{abstract}

\section{Introduction}

Reversible lysine acetylation has been extensively proven to play a critical role in epigenetic regulation of gene expression. $^{1-5}$ Lysine acetylation is catalyzed by histone acetyltransferases (HATs) (writers), leading to transcriptionally active chromatin, while the reverse process is catalyzed by histone deacetylases (HDACs) (erasers) resulting in condensed chromatin and transcriptional silencing. Over the past decades, eighteen mammalian HDACs have been identified and divided into four classes (I-IV) according to their sequence homology to yeast orthologs. ${ }^{6}$ Among them, class I, containing the zinc-dependent HDACs $1-3$ and 8 , is crucial for normal cell survival, homeostasis, proliferation and gene

\footnotetext{
${ }^{a}$ Department of Chemical and Pharmaceutical Biology, Groningen Research Institute of Pharmacy, University of Groningen, Antonius Deusinglaan 1, Groningen $9713 \mathrm{AV}$, The Netherlands. E-mail: f.j.dekker@rug.nl; Fax: +3150 3637953; Tel: +31 503638030

${ }^{b}$ Department of Drug Chemistry and Technologies, 'Sapienza' University, P.le A. Moro 5, 00185 Rome, Italy

'Pasteur Institute, Cenci Bolognetti Foundation, 'Sapienza' University, P.le A. Moro 5, 00185 Rome, Italy

$\dagger$ The authors declare no competing interests.

\$ Electronic supplementary information (ESI) available. See DOI: 10.1039/ c6md00375c
}

expression. ${ }^{7}$ Consequently, mounting evidence points to a link between abnormal HDAC activity and inflammatory ${ }^{8}$ and neurological diseases, ${ }^{9}$ tumorigenesis, ${ }^{9,10}$ and metabolic disorders. $^{11}$

There is a tremendously increasing interest in the development of HDAC inhibitors (HDACi) as potential therapeutic agents for several pathological conditions. To date, a wide variety of small molecules has been reported to target HDACs, with differences in their structure, potency, selectivity and, thus, pharmacological effects. ${ }^{7,12}$ However, a limited number has reached clinical trials and has been approved for medical use, mainly for treatment of cancer patients. ${ }^{13,14}$ The toxicity of the currently available chemotherapeutic agents hamper their further applications. Nevertheless, more selective HDAC inhibitors such as Entinostat targeting only HDAC1-3 is less toxic in clinical trials compared to the non-selective HDAC inhibitors thus providing a perspective towards clinical applications of these inhibitors. ${ }^{15}$ Recently, significant efforts in the field of medicinal chemistry have led to the discovery of more selective inhibitors ${ }^{9,16-21}$ that have shed some light on the role of individual HDACs in specific diseases. Yet, the important question that remains to be answered is whether inhibition of specific HDAC family members enables achievement of the desired biological effects. In order to address this issue it is imperative to develop novel methods to investigate the inhibition pattern needed for each disease model. 
Our interest lies in the better understanding of the role of class I HDACs in inflammatory signaling. It is known that HDACs influence the activation of NF- $\kappa B$ pathway, a key mediator in inflammatory diseases like COPD and asthma. ${ }^{22,23}$ Studies indicate that acetylations of specific lysine residues of the NF- $\kappa \mathrm{B}$ p65 transcription factor affect its transcriptional capacity, duration of action and DNA binding. ${ }^{22,24}$ The alterations observed in $\mathrm{NF}-\kappa \mathrm{B}-$ mediated gene expression after treatment with HDACi confirm a deacetylation-dependent mechanism of activation. ${ }^{25-27}$ Several groups have reported the pivotal role of HDACs 1-3 in inflammatory responses, such as Schwann cell myelination, intestinal epithelial cell homeostasis and allergic reaction. ${ }^{28-32}$ Recently, we explored the function of HDACs 1-3 in inflammatory gene expression in LPS/IFN $\gamma$-stimulated murine macrophages by si-RNA-mediated downregulation. ${ }^{26}$ Our data suggested a proinflammatory role for HDAC1 and 3. Furthermore, selective pharmacological HDAC3 inhibition by RGFP966 demonstrated an attenuation of pro-inflammatory signals in models for inflammatory lung diseases. In another study, HDACi highly potent against HDAC3 and/or HDAC6 have been shown to downregulate mRNA expression of pro-inflammatory cytokines (IL-1 $\beta$ and IL-6) and to promote the transcription of IL-10 gene in $\mathrm{KB} 31, \mathrm{C} 2 \mathrm{C} 12$, and 3T3-J2 cell lines, without affecting the cell viability. ${ }^{33}$ Yet, further optimization is required in the development of novel HDACi, mainly against HDAC1 and 3, as anti-inflammatory therapeutics in order to obtain a clearer picture on which HDAC family member to target.

A very promising approach towards this purpose is the use of chemical epigenetic tools, where a parental clinically available agent is slightly modified to generate closely related inhibitors with a different selectivity profile among biological family members. This approach aims at the fine-tuning of the selectivity of inhibitors over specific family members with respect to their pharmacological effects. ${ }^{34}$ Therefore, we set out to design closely related analogues of Entinostat (MS275), an ortho-aminoanilide containing HDAC1-3 inhibitor with proven therapeutic properties, currently undergoing clinical trials for treatment of various cancers. ${ }^{35}$ These analogues embody isomeric ortho-aminoanilide-type HDAC inhibitors with an azobenzene cap. Inhibition and kinetic studies against class I HDACs were performed in order to evaluate their potency and binding kinetic parameters. Next, we tested the effects of the inhibitors on transcriptional activity and intracellular localization of $\mathrm{NF}-\kappa \mathrm{B}$ p65 as well as inflammatory gene expression in LPS/IFN $\gamma$-stimulated murine macrophages. Interestingly, one analogue (compound 4, para isomer) demonstrated anti-inflammatory characteristics providing insight in the selectivity profile required to attenuate macrophage-mediated pro-inflammatory responses.

\section{Results and discussion}

\section{Chemical epigenetic design}

For our purposes, we chose Entinostat as a starting point to design novel HDACi with refined characteristics. The orthoaminoanilide group, that serves for zinc binding, is advanta- geous compared to the hydroxamic acid moiety (like suberanilohydroxamic acid, SAHA, pan-HDACi) as it assures for selective inhibition among class I HDACs 1-3, reducing cytotoxicity. ${ }^{36-38}$ Our modifications were limited to the cap region of Entinostat, where an azobenzene group was introduced to a para, meta and ortho position with respect to the amine group of the connection unit of the parental inhibitor (compounds 4, 7, and 9 respectively, Fig. 1). Azobenzenes are often used in photopharmacology as photoswitches that allow for two isomeric forms, preferably the cis being more potent than the trans analogue. ${ }^{39-41}$ Previously, we developed successfully SAHA photoswitchable derivatives as potential antitumor agents. ${ }^{42}$ Our data demonstrated that substitution of the cap of the original drug with an azobenzene group in the para, meta and ortho position led to notable differences in terms of potency of the trans form (meta and ortho isomers more potent than para). Additionally, para and ortho trans isomers provided an improved selectivity towards HDACs 1 and 3. These findings encouraged us to employ the same structural alterations in Entinostat in an attempt to improve potency and specificity among HDACs 1-3 and gain useful information on the selectivity profile required to inhibit proinflammatory gene expression in macrophages.

\section{Synthesis}

The starting materials were synthesized as described previously. ${ }^{42}$ Two synthetic routes were followed for the production of the Entinostat analogues (see ESI synthesized by a reductive amination reaction between methyl-4-formylbenzoate and (E)-4-(phenyldiazenyl)aniline. Subsequent hydrolysis to the corresponding ester allowed for coupling to the mono tert-butyloxycarbonyl-protected 2-aminoaniline. Boc deprotection using a solution of $4 \mathrm{~N} \mathrm{HCl}$ afforded compound 4. As for compounds 7 and 9, the peptide coupling between the mono Boc-protected 2-aminoaniline

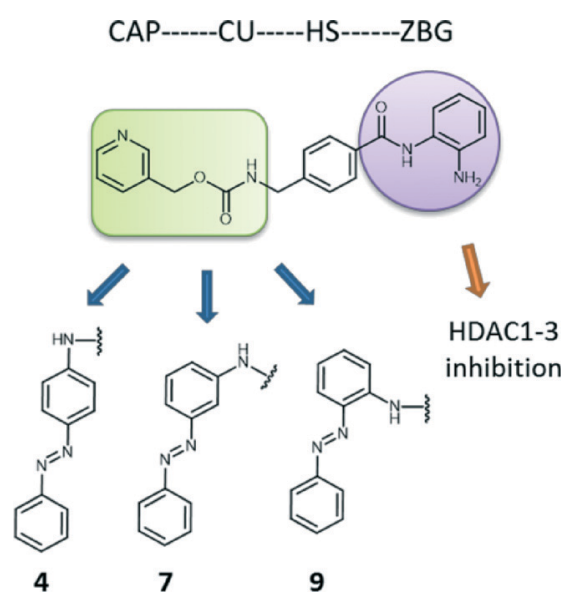

Fig. 1 Design of structural analogues of Entinostat. An azobenzene moiety was introduced into the CAP region in para (4), meta (7) and ortho (9) position in respect to the amine group of the $\mathrm{CU}$ of Entinostat. CU; connection unit, HS; hydrophobic spacer, ZBG; zincbinding group. 
and 4-formylbenzoic acid was performed first followed by reductive amination using the corresponding (phenyldiazenyl) aniline. For compound 7, the deprotection of Boc group was performed as for compound $\mathbf{4}$, while the same conditions led to benzyl cleavage due the basic nature of the amino group at the ortho position of the azobenzene in compound 9. In this case, milder acidic conditions were used by employing trifluoroacetic acid.

\section{$\mathrm{IC}_{50}$ values reveal limited selectivity among HDACs 1-3}

The resulting collection of structurally related HDACi was tested for HDAC inhibition via an assay based on the measurement of the deacetylation of a pro-fluorogenic substrate. ${ }^{42}$ Prior to testing, the stock solutions of the inhibitors (10 $\mathrm{mM}$ in DMSO) were heated at $60^{\circ} \mathrm{C}$ for $10 \mathrm{~min}$ to ensure occurrence of the azobenzene in its trans isomeric form. Various concentrations of each inhibitor were incubated with HDAC1, 2, 3 or 8 in presence of the substrate Boc-Lys(Ac)$\mathrm{AMC}$ at room temperature. After one hour, the reaction was stopped by the addition of SAHA and trypsin to cleave the deacetylated lysine, resulting in the release of fluorescent 7-amino-4-methylcoumarin (AMC). As expected for orthoaminoanilide-type inhibitors, no inhibition was observed against HDAC8 at concentrations up to $50 \mu \mathrm{M}$. It is known that Entinostat inhibits HDACs 1,2 and 3 with a potency of 0.18, 1.2 and $2.3 \mu \mathrm{M}$ correspondigly. ${ }^{43}$ The $\mathrm{IC}_{50}$ values of our isomeric set of compounds were found to be in the low $\mu \mathrm{M}$ range with no meaningful differences among individual family members (see ESI + Fig. S1). We then set out to perform kinetic studies in order to determine the affinity rate constant $\left(K_{\mathrm{i}}\right)$ values, known to be more representative for the inhibitory potencies.

Binding kinetic analysis reaffirms a non-family memberselective profile

Previous studies have shown that enzyme binding kinetics are more informative on the selectivity and behavior of the inhibitors inside the biological targets. ${ }^{44}$ Therefore, we expanded our investigations with determination of the enzyme kinetics on HDAC1, 2 and 3. There is strong evidence that ortho-aminoanilides are slow, tight-binding inhibitors, following the mechanism of enzyme inhibition. ${ }^{34,45}$ In contrast to hydroxamate-based inhibitors, they act via a slow on/slow off binding; they are characterized by low association rate constants $\left(k_{\text {on }}\right)$ and form a tight complex with the enzyme (EI), exhibiting long residence times. In order to determine the kinetic parameters of each inhibitor, Lys-C peptidase was used as a developer instead of trypsin as described by Chou et al. to prevent enzyme degradation. ${ }^{46}$ Moreover, the deacetylation reaction was not stopped by the addition of SAHA but HDAC activity was monitored over time to generate progress curves allowing for the investigation of the rate constants. ${ }^{46}$

According to a previously reported protocol, the measurement of the association rate constants $\left(k_{\text {on }}\right)$ involved the incubation of HDACs 1-3 with different concentrations of the inhibitors in presence of Lys-C and high amounts of the substrate $\left(5 \times K_{\mathrm{M}}\right){ }^{46}$ The fluorescence was monitored for $60-90$ $\mathrm{min}$ at room temperature. The progress curves of all the inhibitors confirmed a time-dependent mechanism of HDAC inhibition and a linear relationship between $k_{\text {obs }}$ and inhibitor concentration as observed before (Fig. S2, $\downarrow k_{\text {on }}$ values were calculated as described in the ESI section). ${ }^{46}$ The offrate constants $\left(k_{\text {off }}\right)$ were determined after 100-fold dilution of samples containing high concentrations of HDACs 1-3 pre-incubated for $1 \mathrm{~h}$ with each inhibitor, and subsequent measurement of HDAC activity as described above. ${ }^{46}$

In terms of selectivity among family members, all compounds appeared to bind faster in HDAC1, less fast in HDAC2 and even slower in HDAC3 (compound 7 presented similar $k_{\text {on }}$ values in HDAC2 and 3) (Fig. 2, ESI + Table S1). More specifically, regarding HDAC1, compounds 4 and 7 were found to bind slightly faster than the reference Entinostat whereas the observed $k_{\text {on }}$ values in HDAC2 were lower. Compound 9 displayed the smallest $k_{\text {on }}$ values in all HDACs 1-3 (even 3.5× slower than Entinostat in the case of HDAC3). A certain pattern was also noticed during dissociation experiments, where we discovered that Entinostat and its azobenzene analogues are generally released rapidly from HDAC3, less fast from HDAC1 and remain longer in HDAC2 (compound 7 presented similar $k_{\text {off values in HDAC1 and 3) }}$ (Fig. 2). Compound 4 showed longer residence in HDAC1 and 3 than compounds 7 and 9, whereas the dissociation rate from HDAC2 was found to be similar for all the developed inhibitors. We then used the $k_{\text {on }}$ and $k_{\text {off }}$ values of the analogues to calculate the affinity rate contants $K_{\mathrm{i}}$, using the

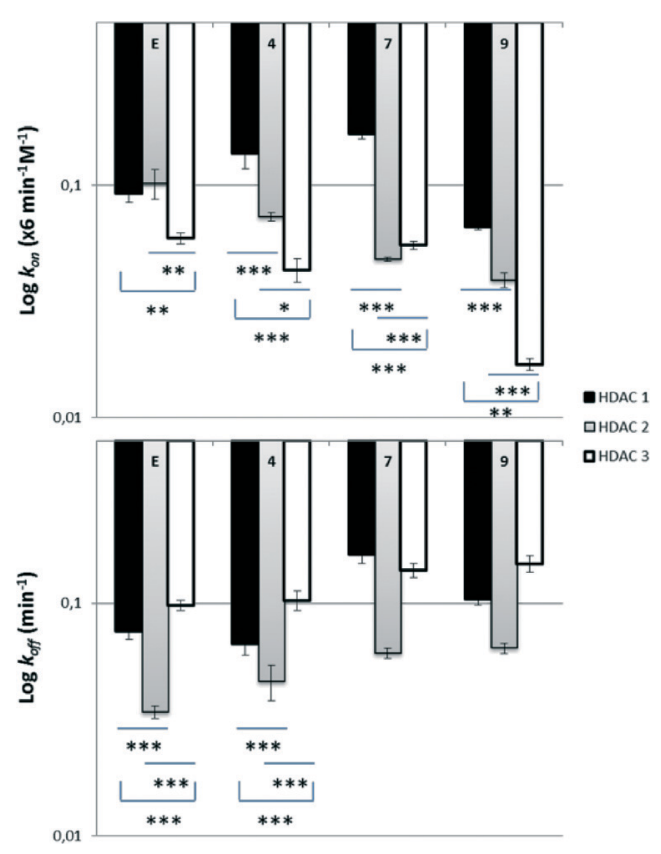

Fig. 2 Comparison bar graphs of the kinetic values of Entinostat (E) and its para (4), meta (7) and ortho (9) azobenzene analogues on HDACs 1-3 expressed in logarithmic scale. The data are presented as mean values of three independent measurements with their respective standard deviations. ${ }^{*} p<0.05,{ }^{* *} p<0.01{ }^{* * *} p<0.001$. 

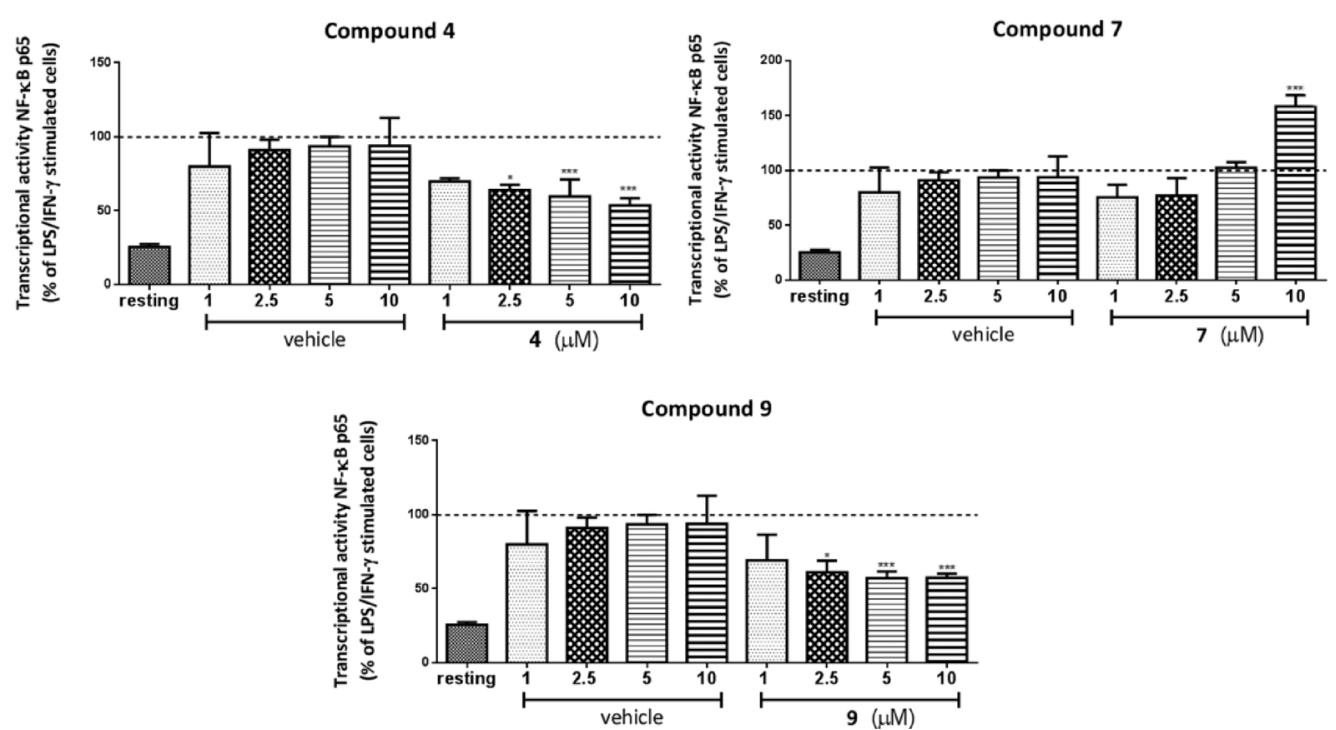

Fig. 3 Effects of para (4), meta (7) and ortho (9) azobenzene analogues of Entinostat on LPS/IFN $\gamma$-induced NF-kB p65 transcriptional activity. RAW blue cells were treated with the respective HDAC inhibitors at the indicated concentrations for $20 \mathrm{~h}$ and stimulated with LPS/IFN $\gamma$ for the last $4 \mathrm{~h}$ of the experiment. For vehicle treatment, cells were pre-treated with a proportional dilution of the inhibitor solvent DMSO. Data are shown as representative data set of 3 independent experiments with the respective standard deviations. $* p<0.05, * * * p<0.001$ compared to vehicle-treated group.

following equation: $K_{\mathrm{i}}=k_{\text {off }} / k_{\text {on }}$ (see ESI, + Table S2). All compounds exhibited statistically significant differences in their potencies against HDACs 1 and 2 in comparison to HDAC3, while stronger inhibition was observed for compound 4 .

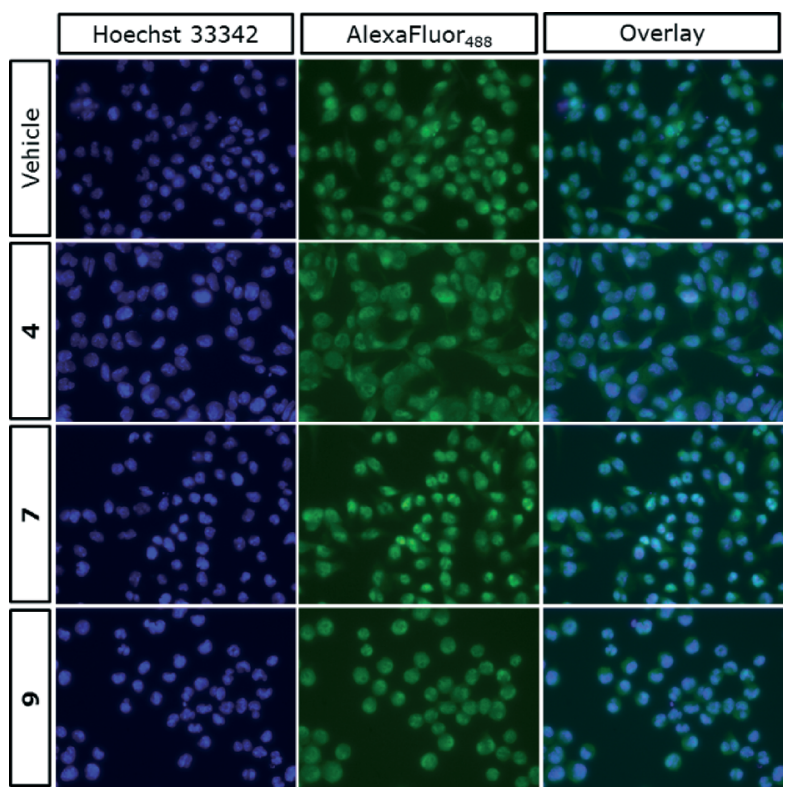

Fig. 4 Effects of para (4), meta (7) and ortho (9) azobenzene analogues of Entinostat on nuclear translocation profile of NF-kB p65 in RAW264.7 macrophages. After $20 \mathrm{~h}$ incubation with $1 \mu \mathrm{M}$ of the respective inhibitors followed by $1 \mathrm{~h}$ LPS/IFN $\gamma$ stimulation, RAW264.7 macrophages were prepared for immunofluorescence microscopy. Green signal represents NF-kB p65 protein, while the blue signal visualizes the Hoechst stained nuclei. The presented data set shows representative images of 2 independent experiments, original magnification $400 \times$. All images were taken under identical instrumental conditions.
However, the differences in potencies among family members are still subtle in order to be further useful to explain any pharmacological variations. The subtle differences in these cases are clearly different from the 10-fold preference for HDAC3 inhibition observed for ortho-aminoanilide type HDAC inhibitors lacking the phenyl group in the linker region. ${ }^{46}$

Compound 4 exhibits anti-inflammatory effects in LPS/IFN $\gamma$ stimulated murine macrophages

Extensive literature has established the connection between HDACs 1-3 and inflammatory signaling in macrophages. ${ }^{47-49}$ In order to investigate the effect of 1-3 selective HDAC inhibitors in inflammatory pathways, we performed a series of pharmacological studies in RAW264.7 murine macrophages. Each time, cells were incubated with the respective inhibitor for $20 \mathrm{~h}$ followed by inflammatory stimulation with LPS and IFN $\gamma$ during the last $4 \mathrm{~h}$ to ensure activation towards M1 (pro-inflammatory) macrophages. ${ }^{50}$

Compound 4 attenuates NF-кB p65 transcriptional activity. Upon stimulation (e.g. by inflammatory cytokines), the p50-p65 heterodimer is released from the complex with $\mathrm{I} \kappa \mathrm{B} \alpha$ and translocated to the nucleus resulting in the upregulation of the expression of specific genes. ${ }^{22}$ As mentioned in the introduction, acetylation and, consequently, deacetylation are of key importance in the regulation of NF- $\kappa \mathrm{B}$ p65 transcriptional activity. The latter was assessed using a reporter gene assay (see ESI†). First, we explored the cell viability after treatment with different concentrations of HDACi. Importantly, compounds 4, 7 and 9 showed no effect on cell viability at concentrations up to $50 \mu \mathrm{M}$ (see ESI + Fig. S3). Next to 

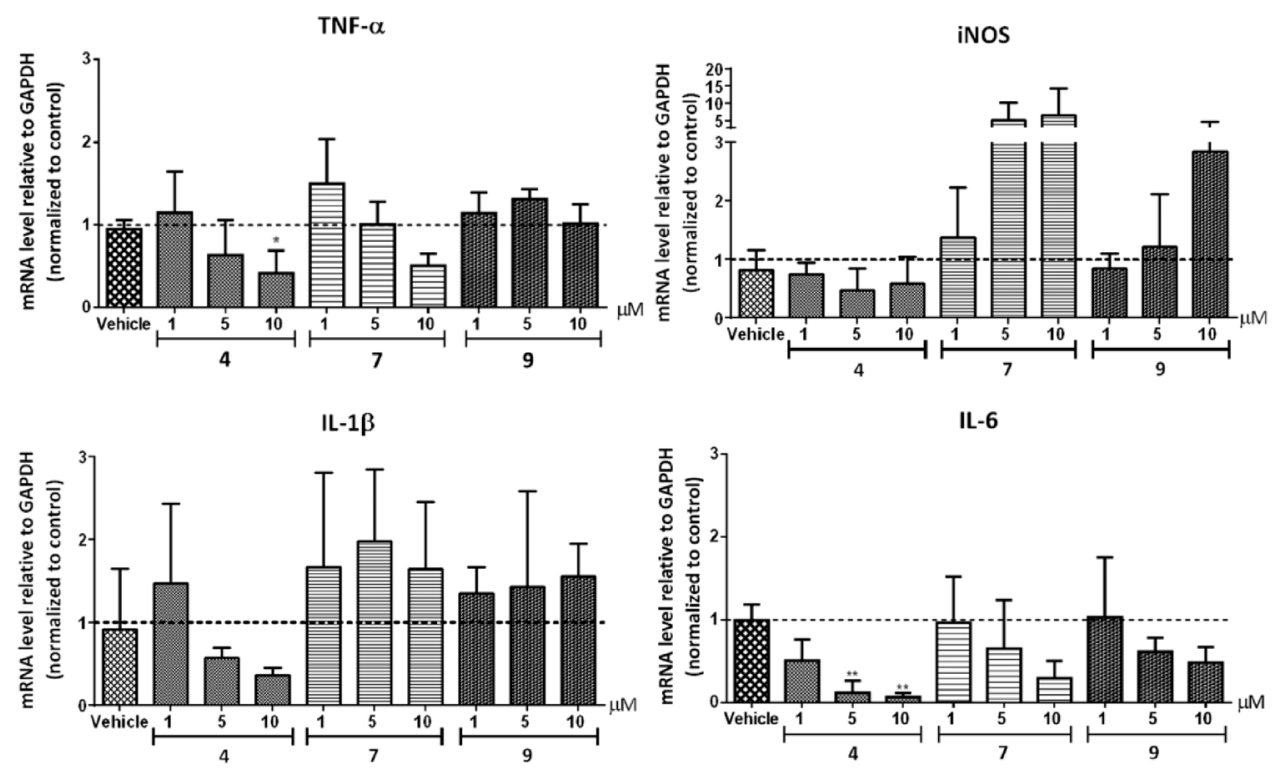

IL-12b
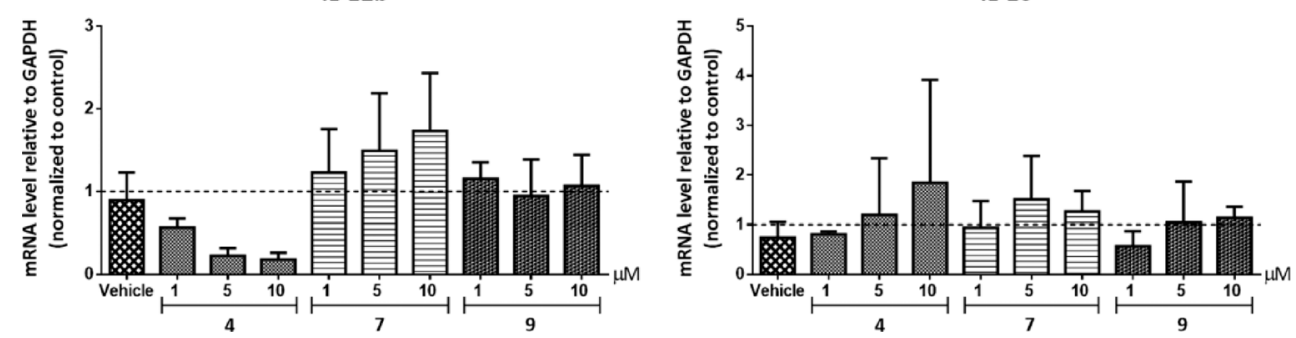

Fig. 5 Effects of para (4), meta (7) and ortho (9) azobenzene analogues of Entinostat on pro- and anti-inflammatory gene expression in RAW264.7 macrophages. Cells were treated with the respective HDAC inhibitors at the indicated concentrations for $20 \mathrm{~h}$ and stimulated with LPS/IFN $\gamma$ for the last $4 \mathrm{~h}$ of the experiment. Gene expression was analyzed by RT-q-PCR. For vehicle treatment, cells were pre-treated with a proportional dilution of the inhibitor solvent DMSO. Data represent the target gene expression normalized to the reference gene and are presented as mean values of 3-4 independent experiments with their respective standard deviations. ${ }^{*} p<0.05,{ }^{*} p<0.01$ compared to vehicle-treated group.

this, the stability of the azobenzene-containing inhibitors towards reduction in cellular environment was previously verified after testing in presence of $10 \mathrm{mM}$ of glutathione. ${ }^{42}$

RAW264.7 cells were treated with compounds 4, 7 and 9 in a concentration range 1-10 $\mu \mathrm{M}$. Interestingly, treatment with para (4) or ortho (9) isomers resulted in inhibition of NF- $\kappa$ B p65 transcriptional activity with the highest effect observed at $10 \mu \mathrm{M}$ (up to $50 \%$ reduction, Fig. 3). On the contrary, a robust activation of NF- $\mathrm{B}$ p65 was found in case of $10 \mu \mathrm{M}$ of meta derivative (7), while lower concentrations gave no change.

These findings provided the first indications that structural modifications and relatively small changes in inhibitory selectivity among HDACs 1-3 can result in considerable differences in pharmacological performance. Yet, considering that at $10 \mu \mathrm{M}$ the HDAC activity is mostly inhibited, the differences in the biological behavior of the analogues cannot be entirely attributed to HDAC inhibition as other cellular targets may also be involved.

Compound 7 increases NF-kB p65 nuclear translocation in contrast to compounds 4 and 9. Fluorescence microscopy was used to locate NF- $\kappa \mathrm{B}$ p65 in the nucleus of LPS/IFN $\gamma$ stimulated RAW264.7 macrophages (see ESI $\ddagger$ ). After proper staining, NF-אB p65 was shown in green while cell nuclei were visualized in blue (Fig. 4). Immunofluorescence microscopy images were overlaid and compared to vehicle. In our experiments, compound 7 seemed to enhance the nuclear translocation of NF- $\kappa \mathrm{B}$ p65 (Fig. 4), while in contrast, compounds 4 and 9 did not influence or reduced it. Collectively, these data are consistent with the studies on the NF- $\kappa \mathrm{B}$ transcriptional activity that indicated inhibition for compounds 4 and 9 and activation for 7. Hence, considering the rather non-selective profile of these inhibitors over HDAC family members, we suggest that other mechanisms crucial for NFkB p65 activity are also affected.

Compound 4 reduces the expression of pro-inflammatory genes. After cellular LPS/IFN $\gamma$-stimulation and treatment with 1, 5 and $10 \mu \mathrm{M}$ of each Entinostat analogue, we set out to monitor the expression levels of TNF- $\alpha$, iNOS, IL-1 $\beta$, IL- 6 and IL-12b. We found that compound 4 caused a statistically significant dose dependent reduction in the expression of TNF$\alpha$ and IL-6 (IL-6 reduced by $90 \%$ at $10 \mu \mathrm{M}$ ) (Fig. 5). Remarkably, compound 4 had no effect on the iNOS expression levels in contrast to compounds 7 and 9, when used at their highest concentrations. Notifying, suppression of IL-1 $\beta$ and IL-12b was demonstrated only after treatment with compound 4 , 
however these effects were not statistically significant. Favorably, these data clarify a pronounced anti-inflammatory profile for the para isomer $\mathbf{4}$ and add to the previously mentioned scenario that the apparent differences in gene expression cannot be solely explained via HDAC inhibition but also through other targets with a key role in inflammatory pathways.

\section{Conclusions}

In summary, we developed three structural analogues of the clinically-used ortho-aminoanilide type HDAC1-3 inhibitor Entinostat in an attempt to explore the role of class I HDACs in macrophage pro-inflammatory signaling. The compounds exhibited small differences in their HDAC inhibitory profile with a slight preference to HDAC1 and 2. Studies on the effect of the inhibitors on LPS/IFN $\gamma$-induced inflammatory responses in RAW264.7 macrophages revealed that compounds 4 and 9 impaired NF- $\mathrm{NB}$ p65 transcriptional activity and suppressed NF-кB p65 nuclear translocation, while an increase was observed in case of compound 7. These findings suggest that, additionally to overall HDAC inhibition, the designed compounds target other mechanisms that play a role in transcriptional activity of NF- $\kappa \mathrm{B}$ p65. Further investigations of the impact of the analogues on the expression of pro-inflammatory genes corroborated the anti-inflammatory profile of compound 4 .

Our work supports that a non-selective inhibition of HDACs 1-3 offers a promising potential towards the desirable anti-inflammatory characteristics, taking into account that possible off-target effects may be significant as well. This sets the stage for further development and exploration of the contribution of HDAC1, 2 and 3 in drug discovery effort aimed at the development of anti-inflammatory therapeutics based on the ortho-aminoanilide scaffold. Moreover, we demonstrated that chemical epigenetics can be employed as a useful strategy in order to fine-tune the family member(s) selectivity with the ultimate goal to develop inhibitors that possess the desired pharmacological behavior.

\section{Acknowledgements}

This work was financially supported by a VIDI grant (723.012.005) from the Netherlands Organization for Scientific Research and an ERC starting grant (309782) from the European Union to F. J. D. Funds from RF-2010-2318330 and IIT-Sapienza Project grants are also acknowledged by A. M. We also thank W. Szymanski for providing the starting materials for the synthesis of the reported compounds.

\section{References}

1 C. A. Mizzen and C. D. Allis, Cell. Mol. Life Sci., 1998, 54, 6-20.

2 B. M. Turner, BioEssays, 2000, 22, 836-845.

3 M. Grunstein, Nature, 1997, 389, 349-352.

4 G. P. Delcuve, D. H. Khan and J. R. Davie, Clin. Epigenet., $2012,4,5$.
5 E. E. Cameron, K. E. Bachman, S. Myöhänen, J. G. Herman and S. B. Baylin, Nat. Genet., 1999, 21, 103-107.

6 I. V. Gregoretti, Y. M. Lee and H. V. Goodson, J. Mol. Biol., 2004, 338, 17-31.

7 M. Dokmanovic, C. Clarke and P. A. Marks, Mol. Cancer Res., 2007, 5, 981-989.

8 M. R. Shakespear, M. A. Halili, K. M. Irvine, D. P. Fairlie and M. J. Sweet, Trends Immunol., 2011, 32, 335-343.

9 K. J. Falkenberg and R. W. Johnstone, Nat. Rev. Drug Discovery, 2014, 13, 673-691.

10 S. Ropero and M. Esteller, Mol. Oncol., 2007, 1, 19-25.

11 J. N. Sangshetti, N. S. Sakle, M. H. Dehghan and D. B. Shinde, Mini-Rev. Med. Chem., 2013, 13, 1005-1026.

12 S. Minucci and P. G. Pelicci, Nat. Rev. Cancer, 2006, 6, 38-51.

13 M. Mottamal, S. Zheng, T. L. Huang and G. Wang, Molecules, 2015, 20, 3898-3941.

14 A. C. West and R. W. Johnstone, J. Clin. Invest., 2014, 124, 30-39.

15 R. Pili, B. Salumbides, M. Zhao, S. Altiok, D. Qian, J. Zwiebel, M. A. Carducci and M. A. Rudek, Br. J. Cancer, 2012, 106, 77-84.

16 A. V. Bieliauskas and M. K. H. Pflum, Chem. Soc. Rev., 2008, 37, 1402-1413.

17 T. A. McKinsey, J. Mol. Cell. Cardiol., 2011, 51, 491-496.

18 M. Fournel, C. Bonfils, Y. Hou, P. T. Yan, M.-C. TrachyBourget, A. Kalita, J. Liu, A.-H. Lu, N. Z. Zhou, M.-F. Robert, J. Gillespie, J. J. Wang, H. Ste-Croix, J. Rahil, S. Lefebvre, O. Moradei, D. Delorme, A. R. Macleod, J. M. Besterman and Z. Li, Mol. Cancer Ther., 2008, 7, 759-768.

19 J. H. Park, Y. Jung, T. Y. Kim, S. G. Kim, H. S. Jong, J. W. Lee, D. K. Kim, J. S. Lee, N. K. Kim, T. Y. Kim and Y. J. Bang, Clin. Cancer Res., 2004, 10, 5271-5281.

20 J. H. Lee, A. Mahendran, Y. Yao, L. Ngo, G. Venta-Perez, M. L. Choy, N. Kim, W.-S. Ham, R. Breslow and P. A. Marks, Proc. Natl. Acad. Sci. U. S. A., 2013, 110, 15704-15709.

21 P. Gupta, R. C. Reid, A. Iyer, M. J. Sweet, D. P. Fairlie, R. C. Reid, A. Iyer, M. J. Sweet and D. P. Fairlie, Curr. Top. Med. Chem., 2012, 12, 1479-1499.

22 M. Ghizzoni, H. J. Haisma, H. Maarsingh and F. J. Dekker, Drug Discovery Today, 2011, 16, 504-511.

23 F. J. Dekker, T. van den Bosch and N. I. Martin, Drug Discovery Today, 2014, 19, 654-660.

24 L. F. Chen, W. Fischle, E. Verdin and W. C. Greene, Science, 2001, 293, 1653-1657.

25 T. van den Bosch, A. Boichenko, N. G. J. Leus, M. E. Ourailidou, H. Wapenaar, D. Rotili, A. Mai, A. Imhof, R. Bischoff, H. J. Haisma and F. J. Dekker, Biochem. Pharmacol., 2015, 102, 130-140.

26 N. G. J. Leus, P. E. van der Wouden, T. van den Bosch, W. T. R. Hooghiemstra, M. E. Ourailidou, L. E. M. Kistemaker, R. Bischoff, R. Gosens, H. J. Haisma and F. J. Dekker, Biochem. Pharmacol., 2016, 108, 58-74.

27 V. Quivy and C. Van Lint, Biochem. Pharmacol., 2004, 68, 1221-1229.

28 B. P. Ashburner, S. D. Westerheide and A. S. Baldwin Jr., Mol. Cell. Biol., 2001, 21, 7065-7077. 
29 Y. Chen, H. Wang, S. O. Yoon, X. Xu, M. O. Hottiger, J. Svaren, K. A. Nave, H. A. Kim, E. N. Olson and Q. R. Lu, Nat. Neurosci., 2011, 14, 437-441.

30 A. Gonneaud, J. M. Gagné, N. Turgeon and C. Asselin, J. Inflammation, 2014, 11, 43.

31 X. Chen, I. Barozzi, A. Termanini, E. Prosperini, A. Recchiuti, J. Dalli, F. Mietton, G. Matteoli, S. Hiebert and G. Natoli, Proc. Natl. Acad. Sci. U. S. A., 2012, 109, E2865-2874.

32 Y. Kim, K. Kim, D. Park, E. Lee, H. Lee, Y. S. Lee, J. Choe and D. Jeoung, J. Biol. Chem., 2012, 287, 25844-25859.

33 R. Di Liddo, S. Valente, S. Taurone, C. Zwergel, B. Marrocco, R. Turchetta, M. T. Conconi, C. Scarpa, T. Bertalot, S. Schrenk, A. Mai and M. Artico, Autoimmunity, 2016, 1-11.

34 F. F. Wagner, M. Lundh, T. Kaya, P. McCarren, Y.-L. Zhang, S. Chattopadhyay, J. P. Gale, T. Galbo, S. L. Fisher, B. C. Meier, A. Vetere, S. Richardson, N. G. Morgan, D. P. Christensen, T. J. Gilbert, J. M. Hooker, M. Leroy, D. Walpita, T. Mandrup-Poulsen, B. K. Wagner and E. B. Holson, ACS Chem. Biol., 2016, 11, 363-374.

35 D. A. Yardley, R. R. Ismail-Khan, B. Melichar, M. Lichinitser, P. N. Munster, P. M. Klein, S. Cruickshank, K. D. Miller, M. J. Lee and J. B. Trepel, J. Clin. Oncol., 2013, 31, 2128-2135.

36 O. M. Moradei, T. C. Mallais, S. Frechette, I. Paquin, P. E. Tessier, S. M. Leit, M. Fournel, C. Bonfils, M. C. TrachyBourget, J. Liu, T. P. Yan, A. H. Lu, J. Rahil, J. Wang, S. Lefebvre, Z. Li, A. F. Vaisburg and J. M. Besterman, J. Med. Chem., 2007, 50, 5543-5546.

37 A. Lu, H. Luo, M. Shi, G. Wu, Y. Yuan, J. Liu and F. Tang, Bioorg. Med. Chem. Lett., 2011, 21, 4924-4927.

38 M. Boissinot, M. Inman, A. Hempshall, S. R. James, J. H. Gill, P. Selby, D. T. Bowen, R. Grigg and P. N. Cockerill, Leuk. Res., 2012, 36, 1304-1310.

39 W. A. Velema, W. Szymanski and B. L. Feringa, J. Am. Chem. Soc., 2014, 136, 2178-2191.

40 F. Lynen and S. Ochoa, Biochim. Biophys. Acta, 1953, 12, 299-314.
41 S. A. Reis, B. Ghosh, J. A. Hendricks, D. M. Szantai-Kis, L. Törk, K. N. Ross, J. Lamb, W. Read-Button, B. Zheng, H. Wang, C. Salthouse, S. J. Haggarty and R. Mazitschek, Nat. Chem. Biol., 2016, 12, 317-323.

42 W. Szymanski, M. E. Ourailidou, W. A. Velema, F. J. Dekker and B. L. Feringa, Chem. - Eur. J., 2015, 21, 16517-16524.

43 N. Khan, M. Jeffers, S. Kumar, C. Hackett, F. Boldog, N. Khramtsov, X. Qian, E. Mills, S. C. Berghs, N. Carey, P. W. Finn, L. S. Collins, A. Tumber, J. W. Ritchie, P. B. Jensen, H. S. Lichenstein and M. Sehested, Biochem. J., 2008, 409, 581-589.

44 F. F. Wagner, Y.-L. Zhang, D. M. Fass, N. Joseph, J. P. Gale, M. Weïwer, P. McCarren, S. L. Fisher, T. Kaya, W.-N. Zhao, S. A. Reis, K. M. Hennig, M. Thomas, B. C. Lemercier, M. C. Lewis, J. S. Guan, M. P. Moyer, E. Scolnick, S. J. Haggarty, L.-H. Tsai and E. B. Holson, Chem. Sci., 2015, 6, 804-815.

45 B. E. L. Lauffer, R. Mintzer, R. Fong, S. Mukund, C. Tam, I. Zilberleyb, B. Flicke, A. Ritscher, G. Fedorowicz, R. Vallero, D. F. Ortwine, J. Gunzner, Z. Modrusan, L. Neumann, C. M. Koth, J. S. Kaminker, C. E. Heise and P. Steiner, J. Biol. Chem., 2013, 288, 26926-26943.

46 C. J. Chou, D. Herman and J. M. Gottesfeld, J. Biol. Chem., 2008, 283, 35402-35409.

47 K. Das Gupta, M. R. Shakespear, A. Iyer, D. P. Fairlie and M. J. Sweet, Clin. Transl. Immunol., 2016, 5, e62.

48 A. M. Grabiec, S. Krausz, W. de Jager, T. Burakowski, D. Groot, M. E. Sanders, B. J. Prakken, W. Maslinski, E. Eldering, P. P. Tak and K. A. Reedquist, J. Immunol., 2010, 184, 2718-2728.

49 M. A. Halili, M. R. Andrews, L. I. Labzin, K. Schroder, G. Matthias, C. Cao, E. Lovelace, R. C. Reid, G. T. Le, D. A. Hume, K. M. Irvine, P. Matthias, D. P. Fairlie and M. J. Sweet, J. Leukocyte Biol., 2010, 87, 1103-1114.

50 A. Mantovani, A. Sica, S. Sozzani, P. Allavena, A. Vecchi and M. Locati, Trends Immunol., 2004, 25, 677-686. 